\title{
Optimal Asymmetric and Maximized Adaptive Power Management Protocols for Clustered Ad Hoc Wireless Networks
}

\author{
Zi-Tsan Chou, Member, IEEE, Yu-Hsiang Lin, and \\ Rong-Hong Jan, Senior Member, IEEE Computer Society
}

\begin{abstract}
IEEE 802.11 is currently the most popular medium access control (MAC) standard for mobile ad hoc networks (MANETs). On the other hand, clustering in MANETs is a promising technique to ensure the scalability of various communication protocols. Thus, we propose an optimal asymmetric and maximized adaptive power management protocol, called OAMA, for 802.11-based clustered MANETs, which has the following attractive features. 1) Given the length of schedule repetition interval (SRI), the duty cycles of both clusterheads and members reach the theoretical minimum. 2) Under the minimum duty cycle constraints, the numbers of tunable SRIs for clusterheads and members reach the theoretical maximum. 3) By means of factor-correlative coterie-plane product, OAMA guarantees bounded-time neighbor discovery between the clusterhead and its member, and between all clusterheads, regardless of stations' individual SRIs and the schedule offset between neighboring stations. 4) The time complexity of OAMA neighbor maintenance is $O(1) .5)$ OAMA adopts a cross-layer SRI adjustment scheme such that stations can adaptively tune the values of SRI to maximize energy conservation according to flow timeliness requirements. Both theoretical analyses and simulation results show that OAMA substantially outperforms existing power management protocols for clustered MANETs, including AQEC [2] and ACQ [14], in terms of duty cycle, adaptiveness, data delay dropped ratio, network lifetime, and end-to-end energy throughput.
\end{abstract}

Index Terms-IEEE 802.11, medium access control, clustered ad hoc network, power management, quorum.

\section{INTRODUCTION}

A mobile ad hoc network (MANET) consists of a set of mobile stations, which are often powered by batteries, without any infrastructure. Due to slow progress in battery technology, the success of MANETs thus relies on energyefficient communications. The radio of a mobile station can be in one of three awake states-transmitting, receiving, and idle listening-or in the doze state. Jung and Vaidya [7] indicated that the power consumption in the idle state is only slightly lower than that in the transmitting and receiving state. Hence to save power, a mobile station has to put itself into the doze state. However, in this state, it cannot transmit nor receive. Thus, the design of a power management protocol, which operates at the medium access control (MAC) layer, becomes critical.

\subsection{IEEE 802.11 Power Management}

IEEE 802.11 [6] is currently the de facto MAC standard for MANETs. As shown in Fig. 1, in 802.11, time is divided into fixed-sized beacon intervals (BIs). Mobile stations operating in the power saving (PS, for short) mode should wake up prior to each target beacon transmission time (TBTT) and wait for a

- Z.-T. Chou is with the Department of Electrical Engineering, National Sun Yat-Sen University, Kaohsiung 804, Taiwan, R.O.C.

E-mail: ztchou@mail.ee.nsysu.edu.tw.

- Y.-H. Lin and R.-H. Jan are with the Department of Computer Science, National Chiao-Tung University, Hisnchu 300, Taiwan, R.O.C.

E-mails: yhlin.cs97g@g2.nctu.edu.tw, rhjan@cis.nctu.edu.tw.

Manuscript received 26 Feb. 2010; revised 15 Oct. 2010; accepted 20 Dec. 2010; published online 11 Mar. 2011.

Recommended for acceptance by V. Misic.

For information on obtaining reprints of this article, please send e-mail to: tpds@computer.org, and reference IEEECS Log Number TPDS-2010-02-0129. Digital Object Identifier no. 10.1109/TPDS.2011.92. random backoff time to contend for broadcasting a beacon frame, which is mainly used for clock synchronization. All PS stations should keep awake during the entire announcement traffic indication message (ATIM) window. If a station $H_{0}$ intends to send buffered data frames to the destination $H_{1}$ currently operating in the PS mode, $H_{0}$ shall first unicast an ATIM frame to $H_{1}$ during the ATIM window. Upon reception of that ATIM frame, the PS destination $H_{1}$ replies an ATIM-ACK to $H_{0}$, and then both $H_{0}$ and $H_{1}$ stay awake for the entire BI. PS stations which neither transmitted nor received an ATIM frame may return to the doze state at the end of the ATIM window. After the ATIM window concludes, station $H_{0}$ sends buffered data to $H_{1}$, and $H_{1}$ then acknowledges its receipts. Note that the transmission and retransmission of ATIM/data frames should follow the distributed coordination function (DCF) procedure. For a more detailed description, refer to [6]. For the ease of reading, the supplement, which can be found on the Computer Society Digital Library at http://doi.ieeecomputersociety. org/10.1109/TPDS.2011.92, provides a list of acronyms and abbreviations used in this paper.

\subsection{Related Work}

From Fig. 1, we can observe that, in 802.11, a PS station should stay awake for the period of ATIM window in every BI, which may incur unnecessary awakeness especially for light-load stations. The first solutions to this problem is [12], which allow PS stations to wake up only for certain BIs. Then, [8], [16] and our previous paper [15] concurrently and independently proposed the similar cyclic quorum-based power management (CQPM for short) protocols to improve the results of [12]. However, [4] indicated that these 


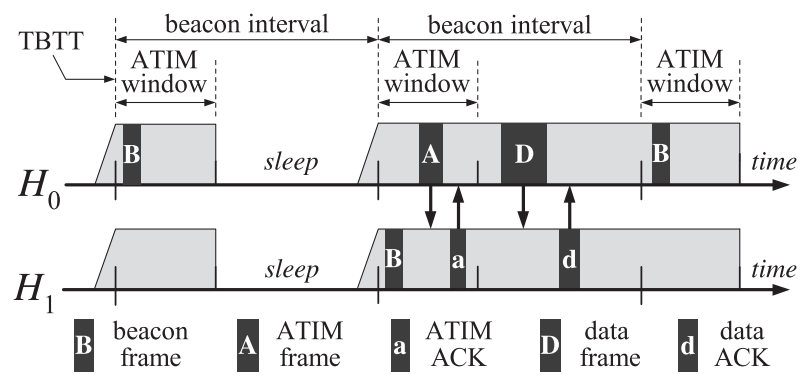

Fig. 1. An example of 802.11 power management operation in MANETs.

protocols [8], [15], [16] require all PS stations must have the same duty cycle (i.e., the fraction of time a station stays awake); otherwise they may completely fail. This implies that they are nonadaptive. Thus, [2] proposed an adaptive CQPM protocol, called Adaptive Quorum-Based Energy Conserving (AQEC). ${ }^{1}$ Referring to Fig. 2, there are two types of beacon intervals in AQEC; one is the awake beacon interval (ABI) and the other is the sleep beacon interval (SBI). The ABI starts with the ATIM window, during which a PS station remains awake and broadcasts its beacon frame. On the other hand, a PS station may doze off during the entire SBI. When a station enters the PS mode, the sequence of BIs is divided into groups such that each group consists of $S$ BIs, where $\sqrt{S}$ is an integer. In each group, the $S$ consecutive BIs are arranged as a $\sqrt{S} \times \sqrt{S}$ grid in a rowmajor fashion. Each PS station can choose one row and one column from a gird of arbitrary size $\sqrt{S} \times \sqrt{S}$ as its ABIs; while the residual BIs are SBIs. We call the group size scheduled repetition interval (SRI for short) since these $S$ consecutive BIs that constitute the specific ABI/SBI pattern repeat regularly. As shown in Fig. 2, by grid-quorum property [2], AQEC guarantees that any two PS neighbors, $H_{0}$ and $H_{1}$, are able to hear each other's beacons (and thus discover each other) in finite time regardless of their schedule offset ${ }^{2} \Delta\left(H_{0}, H_{1}\right)$ and individual SRIs.

Let us define the $A B I$-set $\mathcal{A}(S)$ and ABI-ratio $\alpha(S)$ as the set of the positions of ABIs in an SRI $S$, and the ratio of the cardinality of $\mathcal{A}(S)$ to $S$, respectively. Intuitively, the larger the ABI-ratio, the more frequently the station wakes up, the shorter data reception delay and neighbor discovery time the station may perceive. On the other hand, the smaller the ABI-ratio, the less frequently the station wakes up, the more battery power the station can save. The apparent advantage of an adaptive CQPM protocol is that each PS station can dynamically adjust the value of SRI (and thus the ABI-ratio) according to its residual battery power or other quality-ofservice (QoS) considerations.

Recently, [14] pointed out that, in clustered MANETs [3], there is no need for a quorum-based power management to insist on the overlap property between every pair of PS stations. Referring to Fig. 3, by only guaranteeing the overlap

1. Here, we assume that AQEC performs neighbor maintenance since [4] indicated that, without neighbor maintenance, AQEC may waste significant energy on blindly sending the ATIM frames.

2. With the wide spread of GPS [5] and the availability of industrialstrength clock synchronization mechanisms [17], we assume that the TBTTs of all stations are aligned. After reading Section 2.3, readers can understand that our proposed protocol, called OAMA, can operate in an asynchronous MANET as well. Please notice that the alignment of TBTT does not imply that neighboring stations have no schedule offset.

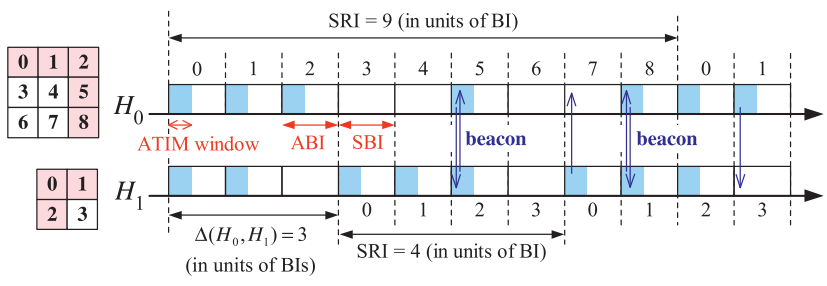

Fig. 2. An example of the neighbor maintenance in AQEC. PS station $H_{0}$ chooses SRI $S=9$ and sets the $j$ th BIs, where $j \in \mathcal{A}(9)=\{0,1,2,5,8\}$, as its ABIs in an SRI. In AQEC, $\alpha(4)=3 / 4$ and $\alpha(9)=5 / 9$.

of ABIs between each cluster member and its clusterhead, and between neighboring clusterheads, the whole MANET can still function well since each member may count on its clusterhead to forward data to the intended destination. On the basis of this principle, Wu et al. [14] proposed the first asymmetric power management (APM for short) protocol, called Asymmetric Cyclic Quorum (ACQ), in which the ABIratio of the cluster member $\alpha_{\mathrm{M}}(S)$ is smaller than that of its clusterhead $\alpha_{\mathrm{H}}(S)$. Since cluster members are the major population in a clustered MANET, this implies that APM may be more energy-efficient than symmetric power management, where both clusterheads and members employ the same ABI-sets construction rules. Specifically, in ACQ, given SRI $S$ and an integer $1 \leq \phi \leq S$, the clusterhead adopts the following rule to build its ABI-set $\mathcal{A}_{\mathrm{H}}(S)$.

$$
\mathcal{A}_{\mathrm{H}}(S)=\left\{0,1, \ldots, \phi-1, s_{1}, s_{2}, \ldots, s_{q-1}\right\},
$$

where $q=\lceil(S+1) / 2 \phi\rceil, \phi-1<s_{1} \leq 2 \phi-1,0<s_{i}-s_{i-1} \leq$ $\phi$, and $s_{q-1} \geq(S-1) / 2$. In contrast, the cluster member in ACQ adopts the following rule to build its ABI-set $\mathcal{A}_{\mathrm{M}}(S)$.

$$
\mathcal{A}_{\mathrm{M}}(S)=\left\{a_{0}, a_{1}, \ldots, a_{p-1}\right\},
$$

where $p=\lceil S / \phi\rceil, a_{0}=0,0<a_{i}-a_{i-1} \leq \phi$, and $0<S-$ $a_{p-1} \leq \phi$. Remark that although APM poses heavier duty cycle on the clusterhead, this problem can be solved by the periodical clusterhead re-election [3].

(a)
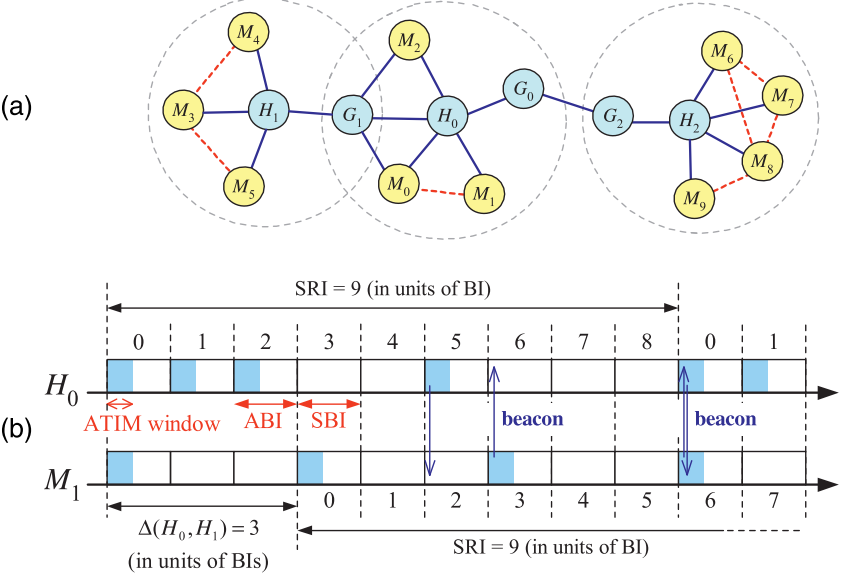

Fig. 3. (a) In clustered MANETs, each station can play one of the following roles: the clusterhead (denoted by $H_{i}$ ), gateway (denoted by $G_{i}$ ), or member (denoted by $M_{i}$ ). The solid blue edge (dotted red edge, respectively) signifies that incident PS stations can (may not, respectively) discover each other in finite time. (b) An example of the neighbor maintenance in ACQ. All PS stations must set the same SRI $S=9$ and $\phi=3$. By fixing $s_{i}-s_{i-1}=a_{i}-a_{i-1}=\phi$, we have $\mathcal{A}_{\mathrm{H}}(S)=\{0,1,2,5\}$ and $\mathcal{A}_{\mathrm{M}}(S)=\{0,3,6\}$. 
Thus, compared with AQEC and ACQ, we can make the following observations: 1) From the viewpoint of adaptiveness, AQEC outperforms ACQ since, given the maximum SRI $S_{\max }$, both the numbers of tunable SRIs for clusterheads and members in AQEC are $\sqrt{S_{\max }}$, while in ACQ, all PS stations must have the same SRI. 2) From the viewpoint of average power consumption, ACQ outperforms AQEC since, under the same SRI $S$, the ABI-ratio of AQEC is about $2 / \sqrt{S}$, while, in ACQ, the minimum ABI-ratios of (clusterhead, member) are about $(\sqrt{2 / S}, \sqrt{2 / S})$ or $(1 / 2,2 / S)$, both of which are, however, larger than the optimal value.

\subsection{Contributions}

Given maximum SRI $S_{\max }$, let us denote the numbers of tunable SRIs for clusterheads and members by $\theta_{\mathrm{H}}\left(S_{\max }\right)$ and $\theta_{\mathrm{M}}\left(S_{\max }\right)$, respectively. We say that an APM is optimal and maximized adaptive if it satisfies the following requirements:

R1. For clusterheads, the number of ABIs in an SRI $S$ is no more than $\lceil\sqrt{S}\rceil+1$. This implies that the ABIratio of the clusterhead is $\alpha_{\mathrm{H}}(S) \approx 1 / \sqrt{S}$.

R2. For cluster members, the number of ABIs in an SRI $S$ is exactly 1 . This implies that the ABI-ratio of the cluster member is $\alpha_{\mathrm{M}}(S)=1 / S$.

R3. $\theta\left(S_{\max }\right)=\theta_{\mathrm{H}}\left(S_{\max }\right) \times \theta_{\mathrm{M}}\left(S_{\max }\right)>\frac{9 S_{\max }^{2}}{16 \ln ^{2} S_{\max }}$.

The reason for $\mathbf{R} \mathbf{1}$ is because [8] proved that, to preserve the overlap property between neighboring PS stations, the number of ABIs in an SRI $S$ can be no less than $\lceil\sqrt{S}\rceil$. The reason for $\mathbf{R} \mathbf{2}$ is because we allow neighboring members can never discover each other. The reason for $\mathbf{R} \mathbf{3}$ is that, under the constraints of $\mathbf{R} \mathbf{1}$ and $\mathbf{R} \mathbf{2}, \frac{9 S_{\max }^{2}}{16 \ln ^{2} S_{\max }}$ is currently the best known asymptotic bound of $\theta\left(S_{\max }\right)$. (See Section 3.)

The major objective of this paper is to design an APM that satisfies R1, R2, and R3. The overall contributions of this paper are as follows:

- In [14], [16], the authors conjectured that the problem of finding an optimal (even nonadaptive) APM schedule for a clustered MANET is NP-complete. We disprove this conjecture by providing a simple yet novel $O(1)$ optimal asymmetric and maximized adaptive power management for the practical value of $S_{\max }$, say 25 . We name our protocol as OAMA. The technical kernel of OAMA is to devise a topologyindependent neighbor maintenance scheme by using the factor-correlative coterie-plane product(defined in Section 2.1) to guarantee the bounded-time neighbor discovery between each clusterhead and its members, and between neighboring clusterheads, regardless of stations' individual SRIs and the schedule offset between neighboring stations.

- Since a PS station may often stay in the doze state, we design the ABI/SBI pattern prediction method such that the sending station in OAMA can predict when its PS neighbor will wake up, thus delivering data frames to it at the right time.

- To illuminate the power of adaptiveness, we design a cross-layer SRI adjustment scheme for OAMA such that PS stations can dynamically tune the values of SRI to maximize power conservation according to flow timeliness requirements.

\begin{tabular}{|c|ccccc|c|}
\hline Clusterhead ABI-Set Table \\
\hline SRI & \multicolumn{7}{|c|}{ Positions of ABIs in an SRI } & ABI-ratio \\
\hline 1 & 0 & & & & 1.000 \\
\hline 2 & 0 & 1 & & & 1.000 \\
\hline 4 & 0 & 1 & 3 & & & 0.750 \\
\hline 5 & 0 & 1 & 3 & & & 0.600 \\
\hline 7 & 0 & 1 & 3 & & & 0.429 \\
\hline 10 & 0 & 1 & 3 & 6 & & 0.400 \\
\hline 14 & 0 & 1 & 2 & 3 & 7 & 0.357 \\
\hline 19 & 0 & 1 & 2 & 6 & 9 & 0.263 \\
\hline 23 & 0 & 1 & 2 & 3 & 7 & 11 \\
\hline
\end{tabular}

\begin{tabular}{|c|c|c|c|c|c|c|c|c|}
\hline \multicolumn{9}{|c|}{ Member SRI-Set Table } \\
\hline SRI & 1 & 2 & 3 & 6 & 11 & 13 & 17 & 22 \\
\hline ABI-ratio & 1.000 & 0.500 & 0.333 & 0.167 & 0.091 & 0.077 & 0.059 & 0.045 \\
\hline
\end{tabular}

Fig. 4. An example of the factor-correlative coterie-plane product stored in a station using table formats. Here, we let $\omega=2$ and $S_{\max }=25$.

- We first theoretically prove the optimality of OAMA. Then, by conducting extensive simulations, we demonstrate that OAMA is much more energyefficient than existing APM protocols [2], [14].

\section{The OAMA Protocol}

OAMA contains three components: a neighbor maintenance procedure, an ABI/SBI pattern prediction method, and a data frame transfer procedure.

\subsection{Neighbor Maintenance Procedure}

Before introducing the OAMA, we need to define the factorcorrelative coterie-plane product.

Definition 1. Given a positive integer $S_{\max }$, let both $\mathcal{S}=$ $\left\{S_{1}, S_{2}, \ldots, S_{m}\right\}$ and $\mathcal{R}=\left\{R_{1}, R_{2}, \ldots, R_{n}\right\}$ be subsets of $\left\{1,2, \ldots, S_{\max }\right\}$. In addition, given $S_{i} \in \mathcal{S}$, let $\mathcal{A}\left(S_{i}\right)=$ $\left\{b_{1}, \ldots, b_{h}\right\}$ be a subset of $\left\{0,1, \ldots, S_{i}-1\right\}$. The Cartesian product of a collection of ordered pairs $\left\{\left(S_{i}, \mathcal{A}\left(S_{i}\right)\right)\right\}$ and a set $\mathcal{R}$ is called a factor-correlative coterie-plane product if it satisfies the following properties: P1) For any integer $t$, we define $t \oplus \mathcal{A}\left(S_{i}\right)=\left\{t+b_{j} \bmod S_{i} \mid\right.$ for all $\left.b_{j} \in \mathcal{A}\left(S_{i}\right)\right\}$. Then, for all $S_{i} \in \mathcal{S}$, we require $\mathcal{A}\left(S_{i}\right) \cap\left(t \oplus \mathcal{A}\left(S_{i}\right)\right) \neq \varnothing$. P2) Given the integer $S_{i}$, let $d_{1}, \ldots, d_{r}$ be the factors (also called divisors) of $S_{i}$. We require $\bigcup_{k=1}^{r} \mathcal{A}\left(d_{k}\right) \subseteq \mathcal{A}\left(S_{i}\right)$ for all $S_{i} \in \mathcal{S}$. P3) Let $\omega=\max \left\{\operatorname{gcd}\left(S_{i}, R_{j}\right) \mid\right.$ for all $S_{i} \in \mathcal{S}$ and $\left.R_{j} \in \mathcal{R}\right\}$. Then, for all $S_{i} \in \mathcal{S}$, we require $\left\{0 \bmod S_{i}, 1 \bmod S_{i}, \ldots, \omega-1 \bmod S_{i}\right\} \subseteq \mathcal{A}\left(S_{i}\right)$.

The neighbor maintenance procedure of OAMA operates as follows: Referring to Fig. 4, every station stores the same two tables: one is the clusterhead ABI-set table $\left\{\left(S_{i}, \mathcal{A}\left(S_{i}\right)\right)\right\}$ and the other is the member SRI-set table $\mathcal{R}$, both of which together form the factor-correlative coterie-plane product. As shown in Fig. 5, a PS station in OAMA can adjust the length of SRI only at the start of each SRI. Once the value of SRI is determined, the PS station playing the role of clusterhead (or gateway) shall consult the clusterhead ABI-set table to set the positions of ABIs and SBIs in the SRI, while the station playing the role of cluster member sets only the zeroth BI as its ABI in the SRI. Fig. 5 depicts an example where the cluster member, $M_{0}$, and clusterheads, $H_{0}$ and $H_{1}$, arrange their individual ABI/SBI schedules according to the OAMA protocol. Note that although 


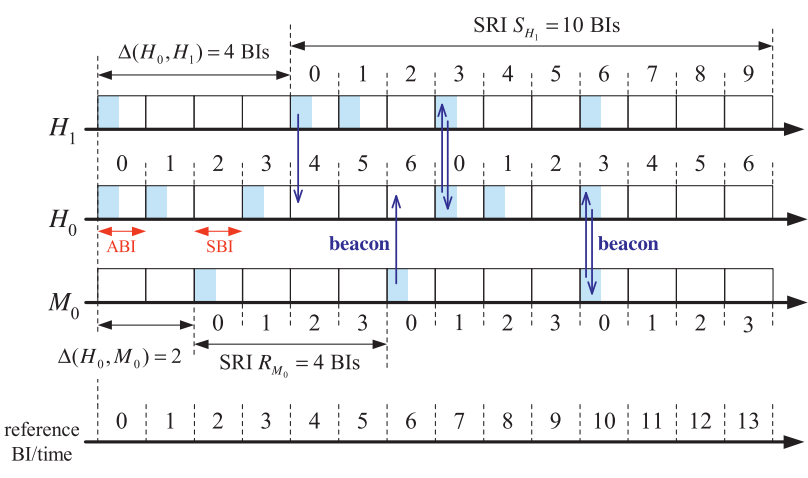

Fig. 5. An example of the neighbor maintenance in OAMA. Here, we assume the schedule offset $\Delta\left(H_{0}, M_{0}\right)$ between $H_{0}$ and $M_{0}$ is 2 (in units of $\mathrm{BI}$ ). According to Fig. 4, PS station $H_{1}$ selects the $\{0,1,3,6\}$ th $\mathrm{Bl}$ as its $\mathrm{ABI}$ within every consecutive $10 \mathrm{Bls}$. Since the factors of 10 include 1 , 2, and 5, OAMA requires $\mathcal{A}(1) \subseteq \mathcal{A}(10), \mathcal{A}(2) \subseteq \mathcal{A}(10)$, and $\mathcal{A}(5) \subseteq \mathcal{A}(10)$.

deriving the optimal tables for $\left\{\left(S_{i}, \mathcal{A}\left(S_{i}\right)\right)\right\}$ and $\mathcal{R}$ may need exhaustive search, the time complexity of OAMA neighbor maintenance is $O(1)$ since it involves only table lookup operations and the table size is constant. Above all, we have the following results. Note that due to space limitations, all the proofs of theorems in the rest of this paper can be found in the supplement, which can be found on the Computer Society Digital Library at http://doi. ieeecomputersociety.org/10.1109/TPDS.2011.92.

Theorem 1. Given bounded $S_{\max }, O A M A$ guarantees that two neighboring clusterheads, $H_{0}$ and $H_{1}$, can discover each other in bounded time regardless of their schedule offset $\Delta\left(H_{0}, H_{1}\right)$ as well as their respective SRIs, $S_{H_{0}}$ and $S_{H_{1}}$.

Theorem 2. Given bounded $S_{\max }$ OAMA guarantees that each cluster member $M_{0}$ and its clusterhead $H_{0}$ can discover each other in bounded time regardless of their schedule offset $\Delta\left(H_{0}, M_{0}\right)$ as well as their respective SRIs, $R_{M_{0}}$ and $S_{H_{0}}$.

\subsection{Data Frame Transfer Procedure}

Since a PS station is not always awake, the sending station must predict when its PS neighbor will wake up. To achieve this goal, each beacon frame shall contain a MAC address, a timestamp, the TBTT of the current BI, the value of SRI, the position of the current $\mathrm{BI}$ in the SRI, and a role-indication bit, besides other 802.11 management parameters. Upon receiving a beacon frame, a station inserts or refreshes the record about this neighbor in its cached neighbor table. Referring to Fig. 6, let $C R T_{H_{0}}$ be the cached record about the TBTT of the $\mathrm{BI}$, during which station $M_{0}$ received the beacon frame from its neighbor $H_{0}$. Moreover, let $I_{H_{0}}$ denote the position of the BI in $H_{0}$ 's SRI $S_{H_{0}}$ in that record. Then at time $t_{1}$, the current position of BI $\delta_{H_{0}}$ in $H_{0}$ 's SRI $S_{H_{0}}$ can be derived via the following formula:

$$
\delta_{H_{0}}=\left\lfloor\frac{\left(t_{1}-C R T_{H_{0}}+I_{H_{0}} \cdot B I\right) \bmod \left(S_{H_{0}} \cdot B I\right)}{B I}\right\rfloor,
$$

where $a \bmod b=a-b\lfloor a / b\rfloor$, if both $a$ and $b \neq 0$ are any real numbers. By using the role-indication bit and comparing $\left(\delta_{H_{0}}, S_{H_{0}}\right)$ with the tables of factor-correlative coterie-plane product, $M_{0}$ can infer whether $H_{0}$ is currently in ABI or SBI.

Once station $M_{0}$ intends to transmit data frames to its PS neighbor $H_{0}, M_{0}$ should first employ the abovementioned $\mathrm{ABI} / \mathrm{SBI}$ pattern prediction method to judge whether $H_{0}$ is

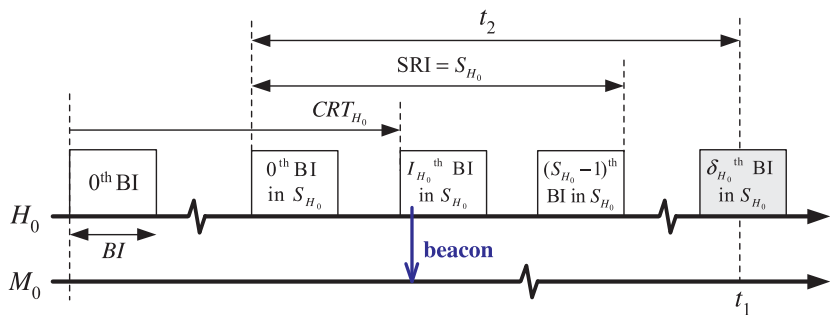

Fig. 6. An example of the ABI/SBI pattern prediction method. We can find that $t_{2}=t_{1}-C R T_{H_{0}}+B I \ldots I_{H_{0}}$, where $B I$ denotes the length of a BI.

currently in ABI or SBI. If $H_{0}$ is currently in SBI, $M_{0}$ should buffer data frames and wait for the coming of $H_{0}$ 's ABI. In $H_{0}{ }^{\prime} \mathrm{S} \mathrm{ABI}, M_{0}$ sends an ATIM frame to $H_{0}$ during the ATIM window. Upon receipt of $M_{0}$ 's ATIM frame, $H_{0}$ replies an ATIM-ACK to $M_{0}$, and both $M_{0}$ and $H_{0}$ remain awake after the close of the ATIM window. Then, $M_{0}$ begins to send data frames to $H_{0}$. When sending data frames to $H_{0}$, if the data queue for $H_{0}$ is not empty, $M_{0}$ will set the more data bit to 1 in the frame control filed [6]. After $H_{0}$ receives the last data frames (with more data bit set to 0 ) from all stations that sent ATIM frames, it immediately returns to the doze state. On the other hand, if $M_{0}$ 's data transmissions for $H_{0}$ cannot be completed within a single BI due to congestion or large amount of buffered data, both $M_{0}$ and $H_{0}$ will remain awake across multiple BIs (some of which may be originally SBIs) until communication is not needed.

\subsection{Synchronization Issue}

The existing CQPM protocols can be classified into two categories: In synchronous mode, CQPM (e.g., [14]) must rely on clock synchronization to guarantee the alignment of TBTT. In asynchronous mode, CQPM (e.g., [16]) requires PS stations to stay awake during the whole period of every ABI, instead of only ATIM window in the ABI, to overcome the alignment problem. Clearly, achieving global synchronization is costly especially in a multihop MANET [12]. Fortunately, OAMA does not require global synchronization. In OAMA, each station tries to synchronize with its neighbors via beacon exchange. Since synchronization in a cluster is easy, OAMA can correctly operate as long as any two neighboring clusterheads can receive each other's beacons. Before looking at how OAMA attains this goal, we need the following theorem:

Theorem 3. Let $\left\{\left(S_{i}, \mathcal{A}\left(S_{i}\right)\right)\right\}$ be the clusterhead ABI-set table in OAMA, where $\mathcal{A}\left(S_{i}\right)=\left\{b_{1}, \ldots, b_{h} \mid b_{j}<b_{j+1}\right.$ for all $1 \leq j \leq h-1\}$. Let $\quad \gamma\left(S_{i}\right)=\max \left\{b_{j+1}-b_{j} \quad\right.$ and $\left.\left(b_{1}-b_{h}\right) \bmod S_{i} \mid 1 \leq j \leq h-1\right\}$. Then, we have $\gamma\left(S_{i}\right) \leq$ $\left\lceil S_{i} / 2\right\rceil$ for all $S_{i} \in \mathcal{S}$.

In OAMA, when a PS station becomes a clusterhead, it temporarily remains awake for $L$ consecutive BIs until it determines the operating (i.e., synchronous or asynchronous) mode. During this period, if that newborn clusterhead received (did not receive, respectively) beacons from its adjacent clusterheads after the close of ATIM windows, it thereafter operates in asynchronous (synchronous, respectively) mode. From Theorem 3, we know that two adjacent ABIs are interspaced by at most $(\lceil S / 2\rceil-1)$ consecutive SBIs, where $S$ is the SRI of a clusterhead. Hence, $L \leq\left\lceil S_{\max } / 2\right\rceil$. Clearly, when a clusterhead operating in 
asynchronous mode can synchronize with all of its adjacent clusterheads, it can then switch to the synchronous mode.

\section{OPTIMALITY OF OAMA}

From the viewpoint of MAC layer, the ABI-ratio, adaptiveness, and average neighbor discovery time are used to judge the goodness of a power management protocol [2], [8], [15]. In this section, we provide performance comparisons among AQEC, ACQ, and OAMA in regard to these metrics.

Theorem 4. Let $\left\{\left(S_{i}, \mathcal{A}\left(S_{i}\right)\right)\right\}$ be the clusterhead ABI-set table in OAMA. We have $\left|\mathcal{A}\left(S_{i}\right)\right| \leq\left\lceil\sqrt{S_{i}}\right\rceil+1$. This implies that the $A B I$-ratio of the clusterhead is $\alpha_{\mathrm{H}}\left(S_{i}\right) \approx 1 / \sqrt{S_{i}}$.

Theorem 5. Let $\left\{\left(S_{i}, \mathcal{A}\left(S_{i}\right)\right)\right\}$ be the clusterhead ABI-set table and $\mathcal{R}$ be the member SRI-set table in OAMA. Moreover, we denote by $\theta_{\mathrm{H}}\left(S_{\max }\right)$ and $\theta_{\mathrm{M}}\left(S_{\max }\right)$ the numbers of tunable SRIs for clusterheads and members, respectively. For $S_{\max } \geq 7$, the adaptiveness of OAMA is $\theta\left(S_{\max }\right)=$ $\theta_{\mathrm{H}}\left(S_{\max }\right) \times \theta_{\mathrm{M}}\left(S_{\max }\right)>\frac{9 S_{\max }^{2}}{16 \ln ^{2} S_{\max }}$.

Combining Theorems 4 and 5 leads to the following result.

Theorem 6. The OAMA is optimal and maximized adaptive.

Then, we investigate the average neighbor discovery time. We define the common awake BIs (CABIs) between stations $H_{0}$ and $H_{1}$ as the reference BIs when both $H_{0}$ and $H_{1}$ are in ABIs. Take Fig. 5 for example, the seventh reference $\mathrm{BI}$ is the CABI between $H_{0}$ and $H_{1}$. Assume that $H_{0}$ and $H_{1}$ select $S_{H_{0}}$ and $S_{H_{1}}$ as their SRIs, respectively. The average neighbor discovery time $\tau\left(S_{H_{0}}, S_{H_{1}}\right)$ between $H_{0}$ and $H_{1}$ is formally defined as follows:

$$
\tau\left(S_{H_{0}}, S_{H_{1}}\right)=\lim _{\Delta t \rightarrow \infty} \frac{\Delta t}{\text { number of CABIs in }[t, t+\Delta t)} .
$$

Theorem 7. Let $\left\{\left(S_{i}, \mathcal{A}\left(S_{i}\right)\right)\right\}$ be the clusterhead ABI-set table in $O A M A$. Then, the average neighbor discovery time between neighboring clusterheads $H_{0}$ and $H_{1}$ is

$$
\tau\left(S_{H_{0}}, S_{H_{1}}\right)=\frac{S_{H_{0}} \times S_{H_{1}}}{\left|\mathcal{A}\left(S_{H_{0}}\right)\right| \times\left|\mathcal{A}\left(S_{H_{1}}\right)\right|} \approx \sqrt{S_{H_{0}} \times S_{H_{1}}} \leq S_{\max } .
$$

Theorem 8. Let $\left\{\left(S_{i}, \mathcal{A}\left(S_{i}\right)\right)\right\}$ be the clusterhead ABI-set table and $\mathcal{R}$ the member SRI-set table in OAMA. Assume that the clusterhead $H_{0}$ and its member $M_{0}$ select $S_{H_{0}}$ and $R_{M_{0}}$ as their SRIs, respectively. Then, the average neighbor discovery time between $H_{0}$ and $M_{0}$ is

$$
\tau\left(S_{H_{0}}, R_{M_{0}}\right)=\frac{S_{H_{0}} \times R_{M_{0}}}{\left|\mathcal{A}\left(S_{H_{0}}\right)\right|} \approx \sqrt{S_{H_{0}}} \times R_{M_{0}} \leq S_{\max }^{3 / 2} .
$$

Fig. 7 depicts theoretical performance comparisons among AQEC, ACQ, and OAMA. Referring (1) and (2), the minimum ABI-ratio of ACQ sensitively depends on the parameter $\phi$. [14] indicated that, given SRI $S$, ACQ has minimum ABI-ratio when $\phi=\lceil\sqrt{(S+1) / 2}\rceil$ or $\lceil(S+1) / 2\rceil$. Hence, we denote by $\mathrm{ACQ}_{1}$ and $\mathrm{ACQ}_{2}$ when ACQ adopts $\phi=\lceil\sqrt{(S+1) / 2}\rceil$ or $\lceil(S+1) / 2\rceil$, respectively. Since ACQ requires all stations, including clusterheads and members,

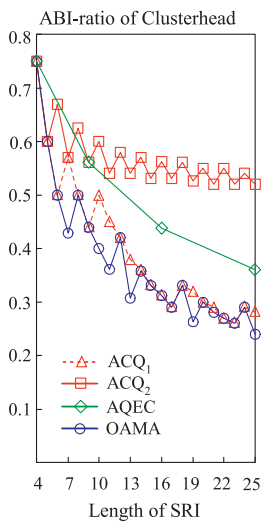

(a)

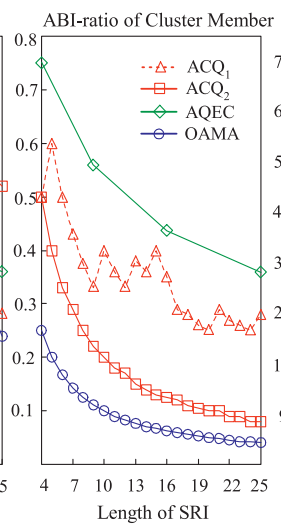

(b)

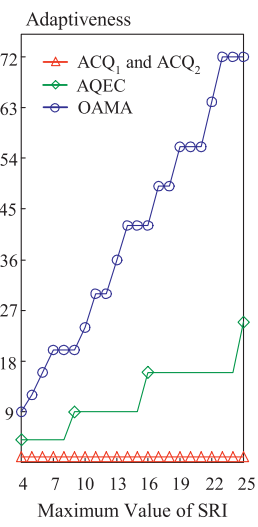

(c)
Fig. 7. The length of SRI versus the ABI-ratio for the (a) clusterhead and (b) member. Part (c) shows the adaptivenesses of various APM protocols.

use the same SRI, its adaptiveness is always 1 . On the other hand, due to the fluctuation of parameters $0<s_{i}-s_{i-1} \leq \phi$ and $0<a_{i}-a_{i-1} \leq \phi$ in (1) and (2), the neighbor discovery time in ACQ is unpredictable.

\section{Cross-Layer SRI Adjustment Procedure}

Before seeing the SRI adjustment procedure of OAMA, we need to derive the maximum one-hop data transfer delay.

Theorem 9. Assume that in a noncongested cluster, a data frame sent from a station to its neighbor can be completed in a single BI, when they are simultaneously awake. Then, in a noncongested cluster, the maximum data frame transfer delay $\xi\left(S_{H_{1}}\right)$ from station $H_{0}$ to its discovered neighboring clusterhead $H_{1}$ that selects $S_{H_{1}} \in \mathcal{S}$ as its SRI is no more than $\left(\left\lceil S_{H_{1}} / 2\right\rceil+1\right) B I-A W$, where $B I$ and $A W$ are the lengths of BI and ATIM window, respectively.

Theorem 10. In a noncongested cluster, the maximum data frame transfer delay $\xi\left(R_{M_{0}}\right)$ from the clusterhead $H_{0}$ to its discovered member $M_{0}$ that selects $R_{M_{0}} \in \mathcal{R}$ as its SRI is $\left(R_{M_{0}}+1\right) B I-A W$, where $B I$ and $A W$ are the lengths of $B I$ and ATIM window, respectively.

Now, we show how to integrate OAMA with a geographic routing protocol, called Greedy-Face-Greedy (GFG) routing [1], so that PS stations along the routing path can adjust the values of SRI in response to the flow timeliness requirement. Theoretically, OAMA, which operates at MAC layer, can integrate with any ad hoc routing protocols. The choice of GFG is mainly because of its simplicity and the freedom of dead-end problem $^{3}$ [1]. We assume that each station piggybacks the residual energy and location information on the beacon frame. In OAMA, the clusterheads and members by default set the SRI values to $\max \{S \mid S \in \mathcal{S}\}$ and $\max \{S \mid S \in \mathcal{R}\}$, respectively. When a clusterhead is aware that the members of its neighboring clusterheads have changed, that clusterhead instantly performs the planarization procedure to ensure the correctness of GFG. When the source station $X$ intends to transmit a

3. The dead-end problem here arises when greedy forwarding fails at a clusterhead that is closer to the destination than all its neighboring clusterheads. 
data flow to the destination $Y$, whose location is known in advanced, it forwards the route request (RREQ) packet specifying its tolerable delay $T_{\text {delay }}$ to the neighboring clusterhead whose residual energy is highest and whose location is closer to the destination. If no such clusterhead can be found, $X$ then enters the perimeter-mode and forwards that RREQ packet to the appropriate clusterhead using the planar face traversal techniques [1].

Upon receipt of the RREQ packet, if the receiving station is not the destination, it appends its address to the RREQ and then propagates that RREQ towards the destination. Assume that RREQ travels from the source $X$, through clusterheads $H_{k_{1}}, H_{k_{2}}, \ldots, H_{k_{n}}$, and finally to the destination $Y$. $Y$ first performs admission control checking whether the following inequality can be satisfied.

$$
\Upsilon=(n+1)(2 B I-A W) \leq T_{\text {delay }}
$$

If not, this means that even all stations along the routing path set SRI $S=1$, the tolerable delay $T_{\text {delay }}$ still cannot be fulfilled. In this case, $Y$ replies the route rejection (RREJ) packet attaching $\Upsilon$ back to the source. The source station can either abort the flow setup or revise the value of $T_{\text {delay }}$ based on $\Upsilon$ and then attempt the above procedure again.

If so, $Y$ should determine the values of $S_{H_{k_{1}}}, \ldots, S_{H_{k_{n}}}$, and $S_{Y}$ such that the following inequality can be satisfied:

$$
\xi\left(S_{H_{k_{1}}}\right)+\cdots+\xi\left(S_{H_{k_{n}}}\right)+\xi\left(S_{Y}\right) \leq T_{\text {delay }} .
$$

It has been proven in [11], [13] that the problem of finding minimum energy routes in a multihop MANET without violating delay constraints is NP-complete. Hence, we design a simple heuristic method to quickly determine the feasible solutions of $S_{H_{k_{1}}}, \ldots, S_{H_{k_{n}}}$, and $S_{Y}$. To balance the power consumption, we hope that all stations along the path use roughly the same SRI. If $S_{H_{k_{i}}}=S_{Y}=S^{*}$ for all $1 \leq i \leq n$, inequality (5) can be reworded as follows:

$$
\left\{\begin{aligned}
(n+1)\left[\left(\left\lceil\frac{S^{*}}{2}\right\rceil+1\right) B I-A W\right] \leq T_{\text {delay }}, & \text { if } Y \text { is a clusterhead, } \\
n\left[\left(\left\lceil\frac{S^{*}}{2}\right\rceil+1\right) B I-A W\right]+\left(S^{*}+1\right) B I-A W \leq T_{\text {delay }}, & \text { if } Y \text { is a clusterhead member. }
\end{aligned}\right.
$$

To maximize $S^{*}$ (and thus, minimize the ABI-ratio), we let

$$
S^{*}= \begin{cases}2\left\lfloor\frac{T_{\text {delay }}-(n+1)(B I-A W)}{(n+1) B I}\right\rfloor, & \text { if } Y \text { is a clusterhead } \\ 2\left\lfloor\frac{T_{\text {delay }}-(n+1)(B I-A W)}{(n+2) B I}\right\rfloor, & \text { if } Y \text { is a member. }\end{cases}
$$

However, the value of $S^{*}$ may not satisfy Definition 1 . Let $S_{\mathrm{H}}=\max _{S \in \mathcal{S}}\left\{S \mid S \leq S^{*}\right\}$ and $S_{\mathrm{M}}=\max _{S \in \mathcal{R}}\{S \mid S \leq$ $\left.S^{*}\right\}$. If $Y$ is a clusterhead, it sets $S_{Y}=S_{\mathrm{H}}$ if its current SRI is greater than $S_{\mathrm{H}}$. If $Y$ is a cluster member, it sets $S_{Y}=S_{\mathrm{M}}$ if its current SRI is greater than $S_{\mathrm{M}}$. Besides, $Y$ replies the route reply (RREP) packet attaching $S_{\mathrm{H}}$ back to the source station in the reverse direction. Every clusterhead along this path changes the SRI value to $S_{\mathrm{H}}$ only when its current SRI is larger than $S_{\mathrm{H}}$. Once the source station received the RREP, it can commence the data flow transmission.

\section{Performance Evaluation}

\subsection{Simulation Model}

We follow the event-driven approach [9] to build a simulator whose architecture is specified in the supplement, which can be found on the Computer Society Digital Library at http://doi.ieeecomputersociety.org/10.1109/ TPDS.2011.92. The simulation area is $700 \times 700 \mathrm{~m}^{2}$. We assume that each station is equipped with a GPS receiver [5], which provides reliable positioning. In a clustered MANET, we adopt GRID [10] as the underlying cluster management scheme. Specifically, the geographic area of the MANET is partitioned into 2D logical grids. Each grid (cluster) is a square area of size $d \times d$, where $d=100 \mathrm{~m}$. The transmission radius of a station is $r=\sqrt{5} d \approx 223.6 \mathrm{~m}$. This ensures that the clusterheads of two adjacent grids are in the transmission range of each other. We assume that only clusterheads can forward packets and clusterheads are reelected every $60 \mathrm{~s}$. With the aid of GPS, we assume that the TBTTs of all stations are aligned. When a station powers on or roams into a new grid, it temporarily sets the SRI value to 1 until it determines its role (clusterhead or member). If that station cannot find a clusterhead within $\left\lceil S_{\max } / 2\right\rceil$ consecutive BIs, it then declares itself (via the roleindication bit in the beacon frame) as a clusterhead. To eliminate the possibility of having multiple clusterheads in a grid, when a station assuming itself as the clusterhead finds another clusterhead having higher residual energy, it silently turns itself as a cluster member. When a clusterhead has aged for $60 \mathrm{~s}$ or leaves its current grid, it appoints the cluster member with the highest residual energy as the new clusterhead and hands over flow-related data to it.

A total of $k$ data flows are established between randomly selected source-destination pairs, where $6 \leq k \leq 12$. Each sender is an ON-OFF Poisson traffic source with interleaved ON and OFF periods of length $10 \mathrm{~s}$ and $15 \mathrm{~s}$, respectively. During the ON period, the average data arrival rate is $\lambda$ Kbps, where $6 \leq \lambda \leq 12$, and the data packet size is 512 bytes. During the OFF period, no traffic is generated. We assume that the channel bit rate is $2 \mathrm{Mbps}$. The lengths of ATIM window and BI are fixed at 25 and $100 \mathrm{~ms}$, respectively. We use $1.65 \mathrm{~W}, 1.4 \mathrm{~W}, 1.15 \mathrm{~W}$, and $0.045 \mathrm{~W}$ as values of power, consumed by the network interface in transmit, receive, idle, and doze state, respectively [7]. The transition between doze state and awake state consumes $0.575 \mathrm{~mJ}$ [7].

In AQEC, each PS station dynamically tunes the value of SRI according to its observed traffic load [2]. Specifically, the SRI adaptation rules of AQEC for both clusterheads and members are as follows:

$$
S= \begin{cases}1, & \text { if traffic } \geq \alpha(1) \cdot \lambda_{\max }, \\ S_{i}, & \text { if } \alpha\left(S_{i+1}\right) \cdot \lambda_{\max } \leq \operatorname{traffic}<\alpha\left(S_{i}\right) \cdot \lambda_{\max } \\ S_{\max }, & \text { if traffic }<\alpha\left(S_{\max }\right) \cdot \lambda_{\max }\end{cases}
$$

where $\lambda_{\max }=12 \mathrm{Kbps}, S_{i}=i^{2}$ for all $i \in\{1,2,3,4,5\}$, $S_{\max }=S_{5}=25$, and $\alpha\left(S_{i}\right)=\left(2 \sqrt{S_{i}}-1\right) / S_{i}$. In ACQ, all stations must use the same SRI and we assume that the value of SRI is 10. Furthermore, in $\mathrm{ACQ}_{1}$, the ABI-sets of the clusterhead and the member are $\{0,1,2,5,9\}$ and $\{0,3,6,9\}$, respectively. In $\mathrm{ACQ}_{2}$, the $\mathrm{ABI}$-sets of the clusterhead and the member are $\{0,1,2,3,4,5\}$ and $\{0,6\}$, respectively.

All simulation runs are carried out for a duration of $1.5 \times$ $10^{9} \mu \mathrm{s}$ and each simulation result is obtained from the average of 10 runs. The confidence level shown in the following figures (except Figs. 9 and 10) is at 95 percent with the confidence interval of $(\bar{U}-1.96 \hat{s} / 3.16, \bar{U}+1.96 \hat{s} / 3.16)$, where $\bar{U}$ is the mean and $\hat{s}$ is the standard deviation of the samples. Note that due to space limitations, the supplement, 


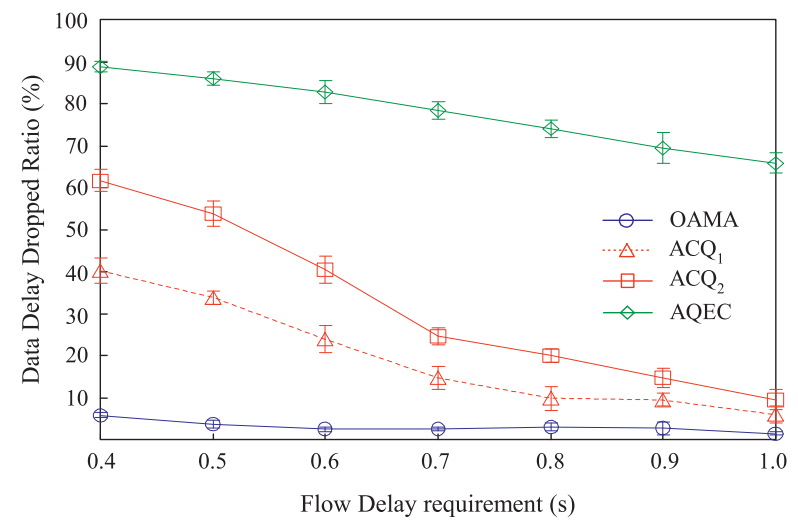

Fig. 8. DDDR versus flow delay requirements. (The total number of stations is 200 and the whole MANET is static. The total number of flows is 9 and $\lambda=9$ Kbps.)

which can be found on the Computer Society Digital Library at http://doi.ieeecomputersociety.org/10.1109/TPDS. 2011.92, provides additional simulation results about the effects of data traffic load, routing protocol, mobility model, station density, etc.

\subsection{Data Delay Dropped Ratio}

The data delay dropped ratio (DDDR for short) is defined as the fraction of dropped data packets caused by violating the end-to-end delay constraints. Fig. 8 shows that when the clustered MANET is static, the DDDR of OAMA can be no more than 6 percent regardless of flow delay requirements. This result justifies the superiority of our adaptive SRI adjustment scheme. The reason for having nonzero DDDR in OAMA is that when a clusterhead, say $H_{1}$, on a routing path needs to reduce the SRI due to the timeliness requirement of another flow, its upstream clusterhead, say $H_{0}$, cannot know the up-to-date ABI/SBI schedule of $H_{1}$ until $H_{0}$ receives a new beacon from $H_{1}$. This will increase the data buffering time at $H_{0}$. Fortunately, Theorem 7 shows that the average neighbor discovery time between neighboring clusterheads is only sublinearly proportional to the SRI. Moreover, Fig. $9 \mathrm{~b}$ depicts that the SRI change rate of a station in OAMA is relatively low. Hence, the DDDR of OAMA is expectably small. The DDDRs of AQEC and ACQ steeply increase as the flow delay requirement decreases. This is mainly because AQEC and ACQ do not perform admission control. However, the DDDR of AQEC is much larger than that of ACQ. The reasons are as follows: In AQEC, stations tune the SRI values according to observed traffic load. Since PS stations do not wake up very often, they can hardly derive the actual arrival rates of the flows. Fig. 9a shows that during the ON periods of a flow, the SRI value of a station oscillates rapidly and sharply. This easily leads to the situation that the upstream clusterhead frequently predicts the wrong $\mathrm{ABI} / \mathrm{SBI}$ schedule of the downstream clusterhead, causing the huge DDDR.

\subsection{Survival Ratio}

The survival ratio is defined as the number of surviving stations (with nonzero energy) over the total number of stations. We assume that the initial energy of every station is 100 Joule. From Fig. 10, we can see that since, in OAMA, the ABI-ratios of both clusterheads and cluster members reach (a)

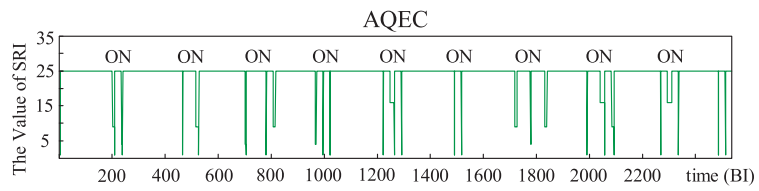

(b)

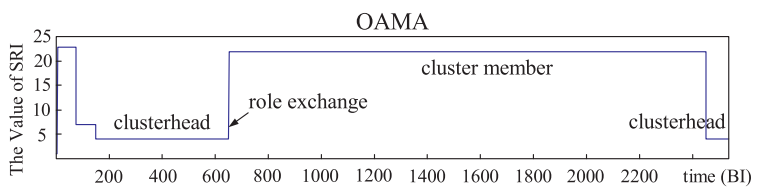

Fig. 9. A snapshot of the evolution of SRI of a station in (a) AQEC and (b) OAMA. (The whole MANET is static and $\lambda=9 \mathrm{Kbps}$. The delay requirement of each flow is $1.0 \mathrm{~s}$.)

the theoretical minimum, the network lifetime of OAMA can be 243,169 , and 225 percent times that of $\mathrm{ACQ}_{1}, \mathrm{ACQ}_{2}$, and AQEC, respectively. Fig. 10 also shows that $A_{C Q}$ has a longer network lifetime than AQEC. This is because $\mathrm{ACQ}_{2}$ has an apparent asymmetric advantage over AQEC (i.e., in $\mathrm{ACQ}_{2}$, a cluster member has a much smaller ABI-ratio than a clusterhead). On the other hand, when the traffic load is light (especially, during the OFF periods), stations in AQEC tend to use the large values of SRI. Besides, a station with maximum SRI in AQEC has a smaller ABI-ratio than a cluster member in $\mathrm{ACQ}_{1}$. Hence, the network lifetime of AQEC can be longer than that of $\mathrm{ACQ}_{1}$.

\subsection{End-to-End Energy Throughput}

The end-to-end energy throughput is defined by dividing the amount of data sent from sources to destinations in flow delay constraints by the total energy consumption of all stations. The authors of [7] pointed out that using energy throughput to judge the goodness of a power management protocol is fairer than using total power consumption since some power management protocols may consume very little energy, but also attain very little throughput. Fig. 11 depicts that the end-to-end energy throughputs of ACQ and AQEC decline with the decrease of the flow delay requirement. This is mainly because both the DDDRs of ACQ and AQEC become large as the flow delay requirement becomes small. On the other hand, thanks to the adaptive SRI adjustment procedure, the end-to-end energy throughput of OAMA can be around $1.6 \mathrm{Kbits} / \mathrm{J}$ regardless of flow delay requirements.

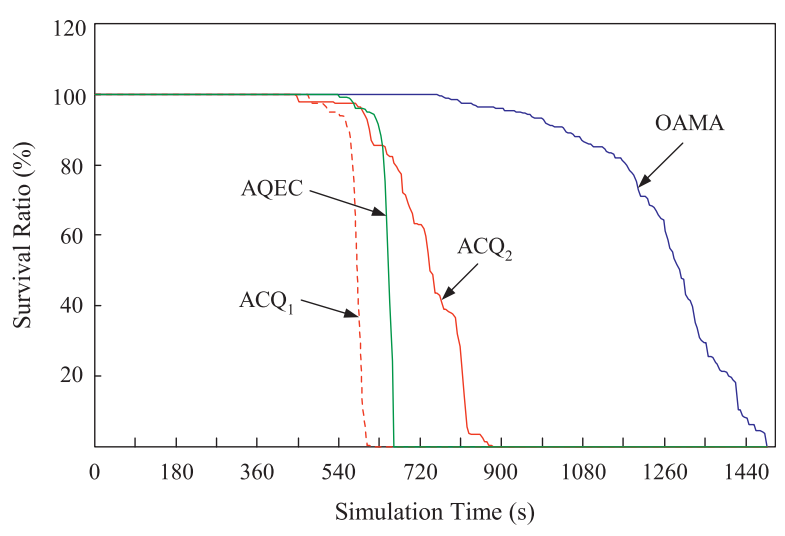

Fig. 10. Survival ratio. (The total number of stations is 300 and the whole MANET is static. The total number of flows is 6 and $\lambda=9$ Kbps. The delay requirement of each flow is $1.4 \mathrm{~s}$.) 


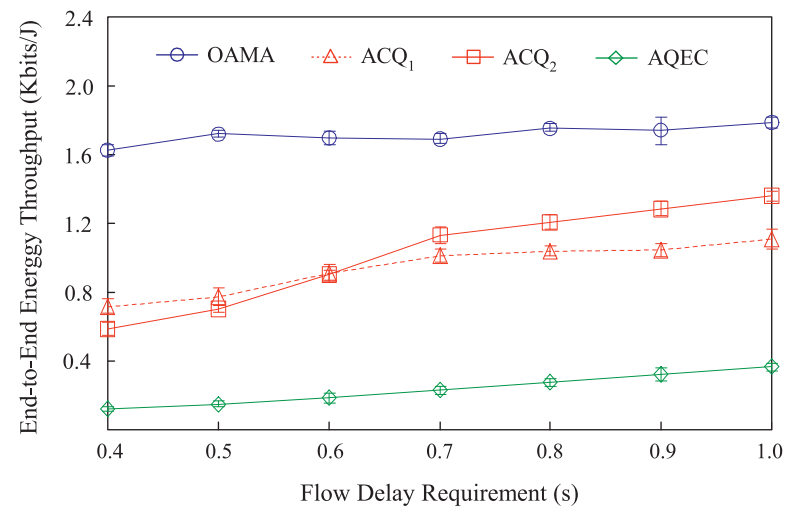

Fig. 11. End-to-end energy throughput versus flow delay requirements. (The total number of stations is 200 and the whole MANET is static. The total number of flows is 9 and $\lambda=9 \mathrm{Kbps}$.)

\section{Conclusion}

IEEE 802.11 has become the de facto MAC standard for a multihop MANET. However, in 802.11, all PS stations should stay awake for the period of ATIM window in every BI. Hence, Chao et al. [2] proposed AQEC, in which each PS station can adaptively tune its SRI according to traffic load. On the other hand, ACQ [14] adopts different ABI-set construction rules for clusterheads and members to earn an asymmetric advantage over AQEC in a clustered MANET. However, in terms of duty cycle and adaptiveness, the performances of AQEC and ACQ are far from optimal. This motivates us to design the OAMA protocol. By means of the factor-corrective coterie-plane product, OAMA ensures the bounded neighbor discovery time. Importantly, OAMA achieves minimum ABI-ratio and maximized adaptiveness for IEEE 802.11based clustered MANETs. Finally, we have proposed a crosslayer SRI adjustment scheme such that PS stations can dynamically adjust the SRI values to maximize energy conservation based on flow delay requirements. Both theoretical analyses and extensive simulations indeed confirm that OAMA significantly outperforms AQEC and ACQ.

\section{ACKNOWLEDGMENTS}

This work was supported by the National Science Council, Taiwan, Republic of China, under grant NSC 99-2221-E110-051.

\section{REFERENCES}

[1] P. Bose, P. Morin, I. Stojmenovic, and J. Urrutia, "Routing with Guaranteed Delivery in Ad Hoc Wireless Networks," Wireless Networks, vol. 7, no. 6, pp. 609-616, Nov. 2001.

[2] C.-M. Chao, J.-P. Sheu, and I.-C. Chou, "An Adaptive QuorumBased Energy Conserving Protocol for IEEE 802.11 Ad Hoc Networks," IEEE Trans. Mobile Computing, vol. 5, no. 5, pp. 560570, May 2006.

[3] B. Chen, K. Jamieson, H. Balakrishnan, and R. Morris, "Span: An Energy-Efficient Coordination Algorithm for Topology Maintenance in Ad Hoc Wireless Networks," Wireless Networks, vol. 8, pp. 481-494, 2002.

[4] Z.-T. Chou, M.-L. Wu, Y.-H. Lin, and R.-H. Jan, "Optimal Fully Adaptive Power Management Protocols for IEEE 802.11 Ad Hoc Wireless LANs," IEEE Int'l Symp. Wireless Comm. Systems, Oct. 2008.

[5] Global Positioning System, http://en.wikipedia.org/wiki/GPS, 2011.
[6] IEEE, IEEE Standard 802.11, Wireless LAN Medium Access Control (MAC) and Physical Layer (PHY) Specifications, Nov. 1999.

[7] E.-S. Jung and N.H. Vaidya, "An Energy Efficient MAC Protocol for Wireless LANs," Proc. IEEE INFOCOM, vol. 3, pp. 1756-1764, June 2002.

[8] J.-R. Jiang, Y.-C. Tseng, C.-S. Hsu, and T.-H. Lai, "Quorum-Based Asynchronous Power-Saving Protocols for IEEE 802.11 Ad Hoc Networks," Mobile Networks and Applications, vol. 10, no. 1/2, pp. 169-181, Feb. 2005.

[9] A.M. Law, Simulation Modeling and Analysis, fourth ed. McGrrawHill, 2007.

[10] W.-H. Liao, Y.-C. Tseng, and J.-P. Sheu, "GRID: A Fully LocationAware Routing Protocol for Mobile Ad Hoc Networks," Telecomm. Systems, vol. 18, no. 1, pp. 37-60, 2001.

[11] M.J. Miller and N.H. Vaidya, "Ad Hoc Routing for Multilevel Power Save Protocols," Elsevier Ad Hoc Networks, vol. 6, pp. 210$225,2008$.

[12] Y.-C. Tseng, C.-S. Hsu, and T.-Y. Hsieh, "Power-Saving Protocols for IEEE 802.11-Based Multi-Hop Ad Hoc Networks," Elsevier Computer Networks, vol. 43, no. 3, pp. 317-337, Oct. 2003.

[13] Y. Wang, X. Liu, J. Ning, J. Yin, and Y. Wu, "Algorithms for Delay Constrained and Energy Efficiently Routing in Wireless Sensor Network," Lecture Notes in Computer Science, pp. 632-642, Springer, Oct. 2006.

[14] S.-H. Wu, C.-M. Chen, and M.-S. Chen, "An Asymmetric Quorumbased Power Saving Protocol for Clustered Ad Hoc Networks," IEEE Int'l Conf. Distributed Computing System, pp. 1-8, June 2007.

[15] S.-L. Wu, P.-C. Tseng, and Z.-T. Chou, "Distributed Power Management Protocols for Multi-Hop Mobile Ad Hoc Networks," Elsevier Computer Networks, vol. 47, pp. 63-85, 2005.

[16] R. Zheng, J. Hou, and L. Sha, "Optimal Block Design for Asynchronous Wake-Up Schedules and Its Applications in Multihop Wireless Networks," IEEE Trans. Mobile Computing, vol. 5, no. 9, pp. 1228-1242, Sept. 2006.

[17] D. Zhou and T.-H. Lai, "An Accurate and Scalable Clock Synchronization Protocol for IEEE 802.11-Based Multihop Ad Hoc Networks," IEEE Trans. Parallel and Distributed Systems, vol. 18, no. 12, pp. 1797-1808, Dec. 2007.

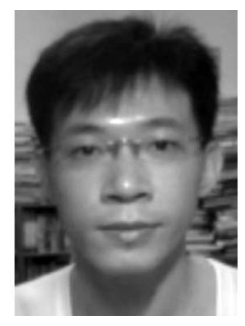

Zi-Tsan Chou (S'98-M'10) received the $\mathrm{PhD}$ degree in computer science and information engineering from the National Taiwan University, in 2003. In 2008, he joined the Department of Electrical Engineering at the National Sun YatSen University, Taiwan, where he is an assistant professor. He holds four United State patents and eight Taiwan patents in the field of wireless communications. His research interests include medium access control, power management, and quality-of-service control for wireless networks. He is a member of the IEEE.

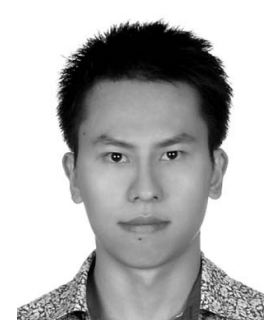

Yu-Hsiang Lin received the MS degree in computer science from the National Chiao Tung University, Taiwan, in 2004, where he is currently working toward the $\mathrm{PhD}$ degree at the Department of Computer Science. His research interests include medium access control and mobility management for wireless networks.

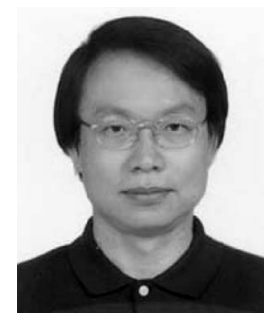

Rong-Hong Jan received the BS and MS degrees in industrial engineering, and the $\mathrm{PhD}$ degree in computer science from the National Tsing Hua University, Taiwan, in 1979, 1983, and 1987, respectively. In 1971, he joined the Department of Computer Science, National Chiao Tung University, where he is currently a professor and an associate dean. His research interests include wireless networks, mobile computing, distributed systems, network security, and network reliability. He is a senior member of the IEEE Computer Society. 